\title{
Educateca: A Web 2.0 Approach to e-Learning with SCORM
}

\author{
Rebeca P. Díaz Redondo, Ana Fernández Vilas, and Jose J. Pazos Arias \\ Department of Telematics Engineering. University of Vigo. 36310 Vigo, Spain \\ \{rebeca, avilas, jose\} @det.uvigo.es
}

\begin{abstract}
This paper introduces the Educateca project, a Web 2.0 approach to e-learning. The project refactors SCORM, the de facto e-learning standard, to embrace the two main shifts in Web 2.0: the WOA (Web Oriented Architecture) and the social trends in user involvement. In this new context of the Internet, flexibility is a must and so big and static e-learning content are no longer effective enough. Thus, we propose a more dynamic approach where small pedagogical units are offered as services to be combine on-the-fly, whenever needed. Social aspects are currently another key and so we propose to adapt the e-learning ecosystem to allow students to innovate and create new content and/or assess the existent one. Clearly, in this new philosophy, recommendation is essential to avoid overwhelming users with too much educative content that are not able to filter, asses and/or consume.
\end{abstract}

Keywords: e-learning 2.0, SCORM, SaaS, Web Service.

\section{Introduction}

E-learning and its related technologies and standards are destined to accept the two main paradigm shifts in the Web 2.0 era [1,2]: the programmable Web and the social Web. In the programmable Web vision, the topology behind WOA (Web oriented Architecture) makes invalid the typical scenario where an organization stores learning materials locally in an LCMS (Learning Content Management System) and deliver them through an LMS (Learning Management System) to the learner's device. Instead, learning organizations should publish and even share their material in the cloud so that learners can access them directly. In the same way, the LMS is becoming another SaaS (Software as a Service) in the cloud so that the student can select a LMS according to the LMS's features and his/her preferences. To sum up, learner, content and LMS are not necessarily in the same domains or from the same providers in a WOA. As the different elements move toward service based approaches, consumers (learners in our case) may opt to mix and match a variety of tools from multiple providers to build an environment that will work best for them.

Apart from these architectonic changes, the Web 2.0 era is characterized by a strong social component. The cloud has to be a place where the learning content is easily generated and published by both teachers and learners so that the collective intelligence of users encourages a more democratic use. 
With the above motivation, this paper introduces the Educateca project, a Web 2.0 approach to e-learning. As it is mandatory in an interoperable environment, standards (Web standards, e-learning standards and mobile standards) are the essential ingredient. Especially in the enhanced learning field, the recognition of needs for standards in e-learning can be tracked back to its origins. Today, ADL SCORM [3] (Shareable Content Object Reference Model) has become the de facto standard to allow content developed within one learning system to be exported and used in all other systems. Despite the fact that SCORM encourages the reusability and remixability in Web 2.0 vision, some aspects of SCORM are not ready to Web 2.0 immersion [4]. With the aim of tackling these shortcomings, Educateca project refactors SCORM infrastructure to provide e-learning in the Web 2.0 arena.

The paper is organized as follows. In the next section we briefly introduce the changes we propose in the SCORM to properly accomplish both social and WOA aspects of the Web 2.0. Section 3 overviews the Educateca vision in the new context of the cloud and in Section 4 we detail the proposed architecture. Finally, conclusions and future work is exposed in Section 5, as well as a comparison between our approach and other works about bringing Web 2.0 to e-learning.

\section{SCORM Shifts to Web 2.0}

In this section we introduce the changes we propose to SCORM content and SCORM LMSs to accomplish the architectonic and social shifts in Web 2.0. First, we include a brief overview about SCORM standard. After having established the SCORM jargon and its essential components, we explain our approach to bringing SCORM closer to both architectonic and social characteristics in Web 2.0.

\subsection{Brief SCORM Overview}

Although SCORM is a vast standard that deals with any aspect related to any $e$ learning aspect, we only focus on SCORM CAM (Content Aggregation Model) and SCORM RTE (Run-Time Environment), the two most relevant components for our approach.

On the one hand, the SCORM CAM defines three main elements used to build a learning experience from learning resources: (1) SCO (Shareable Content Object), a single launchable learning object that utilizes SCORM RTE to communicate with a LMS; (2) Content Organization, a map that represents the intended use of the content through structured units of instruction; and (3) Metadata that should be used to describe these elements in a consistent manner; SCORM strongly recommends the use of the IEEE LOM (see Figure 1). Besides, Content Aggregation is an entity used to deliver both the structure and the resources that belong to a course in a Content Package, which consists of a compressed file with the physical resources of educational content and at least one XML file - called manifest - that embodies a structured inventory of the content of the package: its organization $(<$ organizations $>$ ) and metadata (<metadata $>$ ).

On the other hand, SCORM RTE defines: (1) the launch process as a common way for LMSs to start SCOs; (2) the API as the mechanism for exchanging data between 


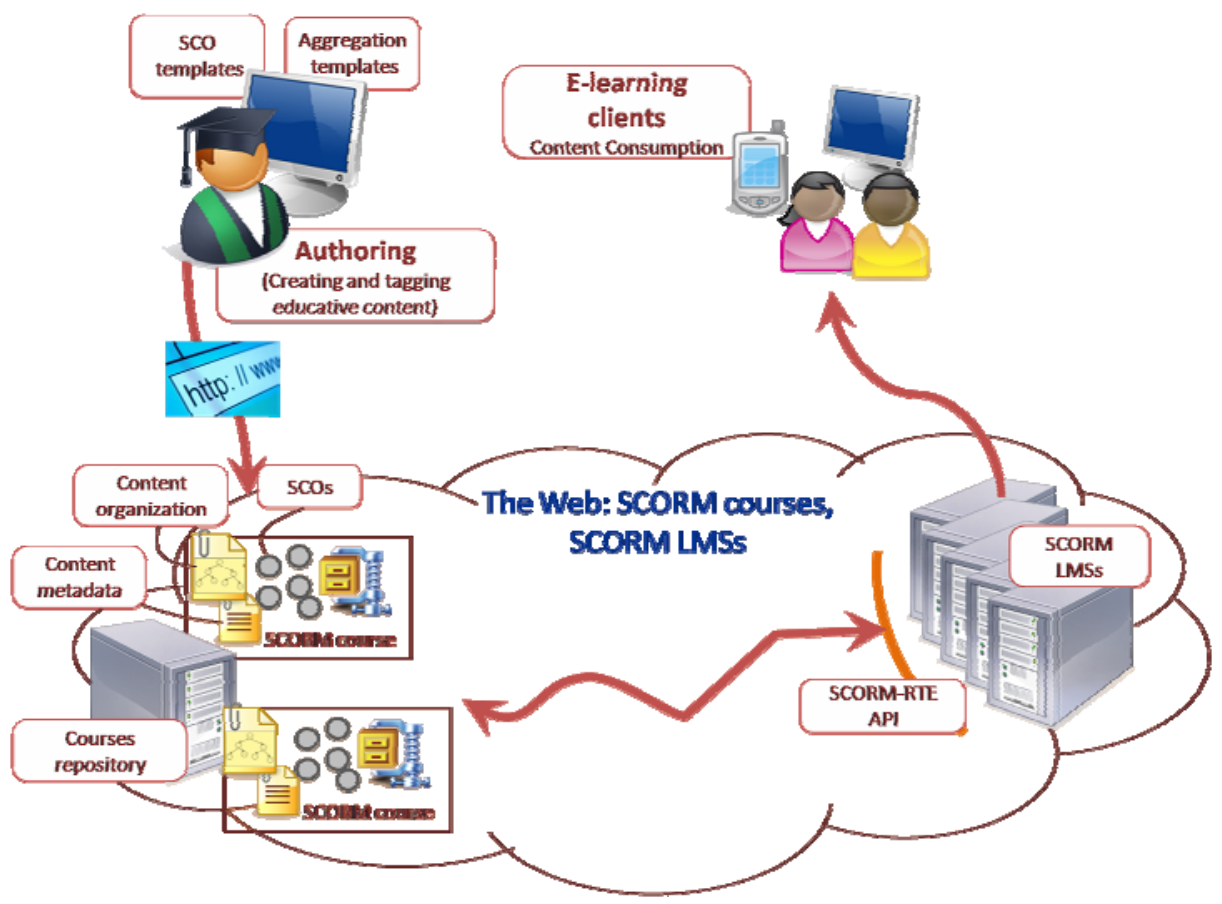

Fig. 1. Typical architecture for any SCORM-compliant e-learning platform

the LMS and the SCO; and (3) a data model as a standard set of elements to define the information tracked for a SCO

\subsection{Architectonic Shift: Driving SCORM to the WOA}

The concept of cloud computing arose as consequence of both the progressive price reduction of consumer electronic devices with computing capabilities and the growing availability of broadband connectivity. Although in origin the cloud was conceived as a way to allow users to access their documents and applications from anywhere and using any device as a lightweight client, this vision has expanded to embrace the socalled Everything as a Service (EaaS or XaaS) trend. Thus, in addition to applications for on-demand use (Software as a Service), the offer encompasses computation, storage, development and communication resources (Platform as a Service, Communication as a Service) and, at the same time, it integrates whichever services may be provisioned through the e-commerce technologies, including human resources in any areas of activity (what we may term User as a Service). Therefore, the cloud lodges arbitrary resources that appear as high-granularity services that may be composed in a flexible manner in response to complex necessities. In this new context, both learning software and content should not be delivered in a packetized way anymore. Instead, we propose to offer these elements as autonomous ones with the aim of being available to be used separately according to the users' needs. 
Therefore, to integrate SCORM content and processes in the WOA cloud we propose the following modifications. First, the compressed file structure of a SCORM course (learning content) would be replaced by set of independent pedagogical units able to communicate to the SCORM RTE to be run (independently or cooperatively) whenever needed. Thus, each SCO becomes an SCS (Sharable Content Service) according to the SaaS conception. Besides, the organization of a SCORM course remains, but it is separated from the learning objects itself. This provides more flexibility to users which finally decide if they prefer a pre-established course definition or an ad-hoc composition of pedagogical units according their interests.

Secondly, the SCORM RTE itself (learning software) is deployed as a set of Web Services offering the SCORM API. Finally, the SCORM CAM (Content Aggregation Model) is replaced by a SAM (Service Aggregation Model), a service (learning software) in the cloud that provides access to a set of SCSs according to a specific organization.

\subsection{Social Shift: Collaborative SCORM}

Social networks appear as a true social and cultural phenomenon on the Internet, whose impact is evident in any social sector. In fact, there are millions of users who already engage in online social interactions as part of their daily lives: sharing information, exchanging experiences and opinions, tagging elements, ratting content and services, even creating or uploading their own content to be shared to other users. Thus, social networks have facilitated a notable change of attitude in users: from a more passive to a really active on. This change is not only restricted to the social networks context, it has spread out to any aspect in the Internet, and e-learning cannot stand aside.

Consequently, we propose an environment where learners have a more leading role in a context where they are no longer passive receivers of information given by a teacher, but active participants whose opinions are took into account. Thus, we revise two main aspects in m-learning. Firstly, describing and cataloguing learning objects is no more responsible for the teaching committee. On the contrary, learners tag and rate learning objects as they use the platform. Secondly, not only tagging but authoring also moves from teachers to learners so that these can create or modify content to contribute to the learning distributed repository. Besides, users can create and share course organizations combining pedagogical units created by them or by any other user.

This vision of providing a suitable space for active learners comes up against the SCORM conception. SCORM was created assuming a single authority (usually a teacher) that creates and assigns metadata for a collective of students. This metadata theoretically allows searching and combining learning objects to make them accessible for other purposes, but, in practice, SCORM content hardly include metadata. Consequently, it is difficult for a community to alter or add metadata tags within SCORM infrastructure, and it is currently impossible for a learner to contribute to a course by adding or altering its content.

Therefore, we propose to change the processes which affect metadata and organization specification to support this environment of participation with SCORMcompatible infrastructures. Apart from distributing authoring (both of learning objects 
and organizations), a Web 2.0 approach requires an open access from students to metadata so that both learning objects and organizations are collaboratively tagged.

\section{Educateca Vision}

Figure 2 depicts the Educateca interpretation of Web 2.0 principles in the e-learning field. One of the aspects that must be highlighted is how the teacher and student roles change in this new model. Whereas in the traditional model (Figure 1) both roles are totally separated, in this new approach students are expected to adopt a prosumer attitude. Thus, students are not more those who receive courses and pedagogical units and whose only obligation is learning the provided content and demonstrate their new knowledge resolving an exam; instead they have now the opportunity of providing new educative content.

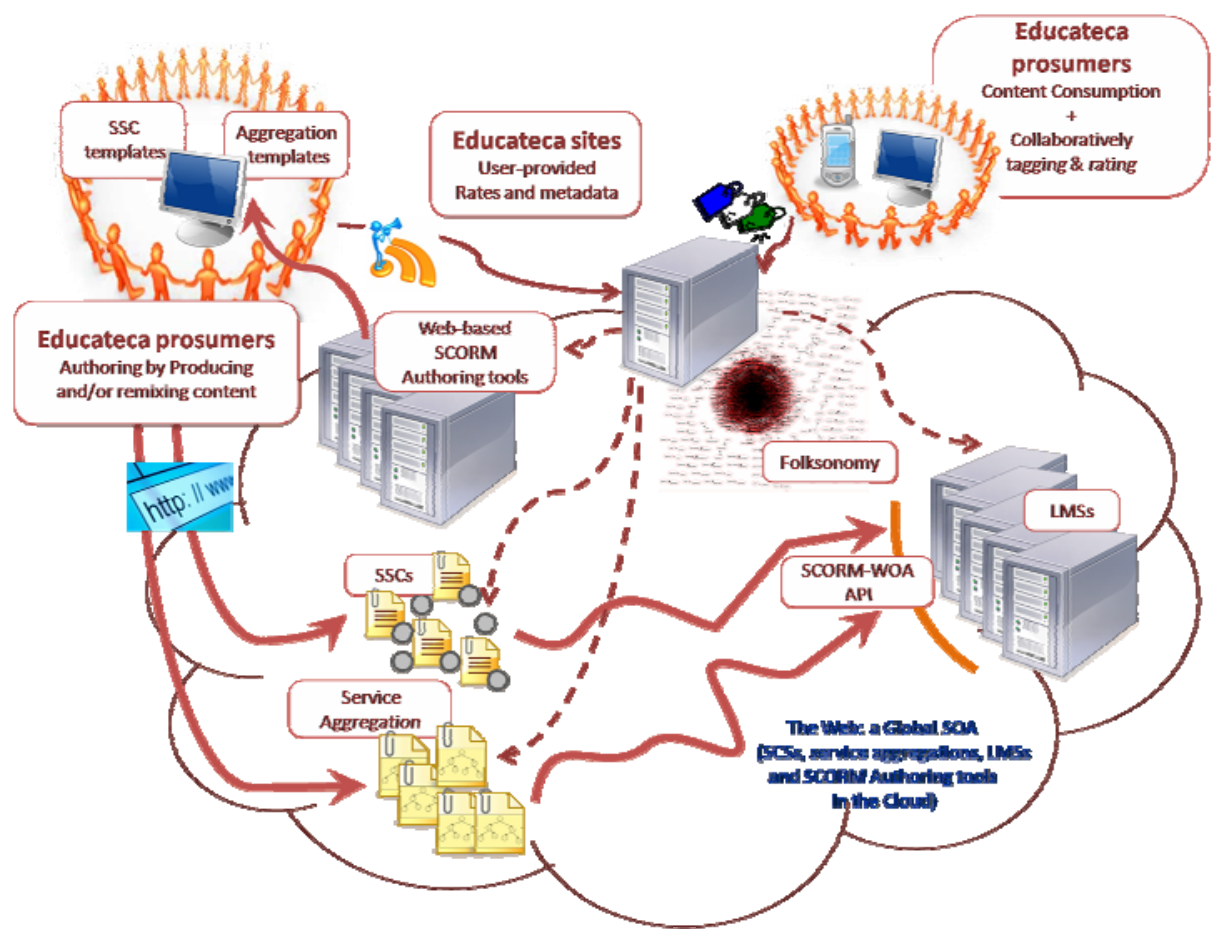

Fig. 2. Proposed architecture for SCORM-compliant e-learning 2.0

According to the WOA interpretation of a SCORM course, their two main components are the SAM (Service Aggregation Model) and SCS (Sharable Content Service), respectively mirrors of CAM and SCO. The CAM service maintains the structure and location of the SCSs in a course but not the SCSs themselves; whereas a SCS consists mainly of an XML template of the learning content, the metadata describing that content and the location of the resources (assets) in the template. 
Consequently, the creation of new educative contents can be done by prosumers in two ways: (i) creating their own pedagogical units (SSCs) and (ii) defining new service aggregations that combine already developed SSCs. With this aim, and according the WOA in the Web 2.0, they may use any SCORM-complaint authoring tool that is offered as a service in the cloud. Teachers, on another hand, continue taking their typical role and, as experts in the field, they create, tag (using metadata) and upload both new pedagogical units as self-content educative services (SSCs) and service aggregations.

The active role of students goes further than creating new elements: prosumers have also the possibility of given their opinion about other SSCs and service aggregations as well as labeling them. Tags given by prosumers allow creating and dynamic updating a folksonomy which support a mechanism to classify and search educative units whenever needed. In fact, users look for pedagogical content according to key-words and their decision of choosing one or another will be likely based on the rates each unit has received. So, instead of having an environment where the quality of content is assumed (like in classical models), this new model entails a certain degree of competition where the opinion of consumers gives a measurement of the quality. Finally, and for courses consumption, users may use any SCORMcomplaint LMS having a Web-based interface, just according to the main SCORM principles that claims for open e-learning environments.

To conclude, Educateca sites maintain information about all the previously mentioned elements: the folksonomy, references to locate SCSs and service aggregations, and references to locate LMSs and authoring tools. Therefore, any prosumer, after login the Educateca system using a Web navigator (in any suitable device: a computer, a mobile phone, etc.), may use the educative environment to create, tag, rate and/or consume pedagogical units in a totally transparent way. Simply the Educateca site recommends the most suitable LMSs to represent properly the educative content according to the user's device.

\section{Details of the Proposed Architecture}

The work introduced in this paper involves the transfer of previous approaches of our research group to the new paradigms in the Web. Being inspired by the personalized e-learning platform T-MAESTRO [5], we have added social and WOA conceptions, central milestones of the Web 2.0. Especially, it is remarkable the following technical aspects in this work. Firstly, users have the possibility of choosing the characteristics they ask to a LMS to operate with or delegate this election to a LMS raking, consequence of third parties' rates. Secondly, it is possible to obtain personalized recommendations of the available content. Given the social nature of our proposal, Educateca joins together formal SCORM metadata (usually provided by expert users, teachers mainly) and informal free-tags (usually given by less expert users, students mainly). Integrating both kind of data entails having a plain structure (folksonomy) which will be the base to establish user's profiles and to run the recommendation algorithm. Thirdly, discovering any element in the proposed architecture over a service philosophy entails redefining SCORM aggregations as BPEL specifications that orchestrate content coming from different SCORM organizations. 


\subsection{Exporting LMs as a Virtual Web Service}

E-learning in the cloud should give the illusion of infinite resources available on demand, eliminating the students need to plan far ahead for e-learning provisioning and following a pay-as-you-go model. With this illusion, infinite LMS would be available in the cloud with different features: cost, efficiency, level of personalization, etc. In the proposed architecture, student delegates the process of discovering and selecting an appropriate LMS to Educateca sites so that some form of SLA (Service Level Agreement) between the student and the Educateca provider has to be defined. Concretely, Educateca sites virtualize LMS by offering a Virtual Web Service, in the sense defined in our previous work in [6, 7], which we call VLMS (Virtual-LMS). Client Device uses the V-LMS as any other service in the cloud and only Educateca sites are aware of the virtual nature of the service and materialize the service in a real LMS by incorporating user-defined preferences, load-balancing, availability, caching, etc.

\subsection{Social Content Discovery and Recommendation}

In order to support e-learning community, Educateca sites incorporate mechanisms to assist the users in a personalized access to e-learning material (discovering and recommendation). To be precise, Educateca strategy is based on collaborative tagging. Folksonomy-like structures are maintained for users and e-learning material in the cloud which support social collaborative filtering for the discovery and recommendation of e-learning material.

The Educateca community collaborate in creating, sharing and describing contents so that students and teachers tag their own contents but also the ones incorporated by others. Each SCS, SAM or even LMS is associated with a set of weighted tags (tagcloud). Similarly, the users in Educateca community have their own tagcloud, representing his/her profile. The user's tagcloud-profile registers the tags of e-learning material he/she has rated and the weights which resume these ratings and so the user's interest. The social collaborative filtering strategy implements an algorithm of tag cloud comparison which is mathematically described in [8].

\subsection{Introducing BPEL in SAMs}

As we have mentioned before, using BPEL4WS in the definition of the SAM components of our model is the natural way of turning an aggregation of SCORM contents (SCOs) into an aggregation of SCORM service (SCSs). As service composition becomes the main concern of the application development process in XaaS paradigm, it is also the case in "e-learning as a service". At this respect, BPEL4WS and its related specifications [9] provide the standard mechanism for defining service compositions in the form of choreographies of Web services, that is, in the form of aggregation of services according to certain rules, e-learning rules in our case. These e-learning rules are the ones defined in SCORM 2004 Sequencing and Navigation specification, derived from IMS Simple Sequencing (IMS SS) specification. Sequencing is what happens when learner exits a SCO, that is, sequencing is responsible for determining what happens next. Further, it orchestrates the flow and status of the course as a whole. This orchestration consists of the sequence definition itself, a set of rules, and the tracking data which records the current state of the elearner activity. 
In brief, our BPEL-style solution to SCORM WOA is as follows. Firstly, the SCORM aggregation is translated into a set of <partnerlink $>$ and <invoke $>$ definitions in BPEL. Secondly, sequencing rules (if-then conditions), limit conditions, constraint choice controls, etc. in a typical SCORM manifest, are translated into BPEL structure activities <sequence $>,<$ flow $>,<$ switch $>,<$ while $>$ and $<$ pick $>$. Finally, tracking data is registered as a set of $<$ variable $>$ elements which are used in the BPEL specification of the course to restrict the BPEL process which implements the course.

Architectonically speaking, the actual process of sequencing SCORM occurs whenever a course is launched, whenever a SCO exits or whenever the learner makes a navigation request through the LMS. For that, a typical SCORM-compatible LMS implements a set of defined algorithms that apply the sequencing rules to the current set of tracking data to determine which activity should be delivered next. Educateca incorporates these algorithms in the LMSs by integrating ActiveBPEL Engine ${ }^{l}$. However, we cannot obviate that content authoring turns into social so that every user in the community should be able to publish a new course organization or a modified version of an existing one. To facilitate this scenario, we provide SAM templates in Educateca sites.

\section{Conclusions}

There are some other approaches in the literature which transfers e-learning platforms to a service-oriented approach and even to cloud e-learning. In [10], Vossen \& Westerkamp discuss typical problems of SCORM-related standards and propose a service-oriented approach as a solution, but any specific architecture or implementation is introduced. In [11,12] Dong proposes the use of cloud computing as a base for modern e-learning by introducing the BlueSky cloud framework which virtualizes physical machines for e-learning systems. Similarly, CoudIA (Cloud Infrastructure and Application) project [13] runs private cloud infrastructure for e-learning and collaboration in the university environment of HFU (Hochschule Furtwangen University). With a lab-oriented application, Virtual Computing Laboratory (VCL) [14] (by North Carolina State University) enables students to reserve and access virtual machines (VMs) with a basic image or specific applications environments, such as Matlab and Autodesk. Meanwhile the above works focus on the infrastructure dimension of cloud-based e-learning, our proposal gives a more ambitious conception for e-learning in the cloud. Not only the infrastructure but the content and the metadata are freely in the cloud and then reassembled on demand by Educateca sites. Apart from that, the social dimension of e-learning in the cloud is considered by turning students and teachers into a peer-to-peer community.

The Educateca project is one more step towards new perspectives for enhanced learning with SCORM. The Educateca site and the online authoring tool are based on our previous work in t-MAESTRO [5], a SCORM-compatible infrastructure for personalized t-learning experiences combining TV programs and learning contents in a personalized way, with the aim of using the playful nature of TV to make learning more attractive and to engage TV viewers in learning. To be precise, we have adapted the functionality and architecture of the t-MAESTRO ITS (Intelligent Tutoring

\footnotetext{
${ }^{1}$ http://www.activevos.com
} 
System) which constructs the t-learning experiences by applying semantic knowledge about the t-learners; and the A-SCORM Creator Tool, the authoring tool which allow teachers to create adaptive courses with a minimal technical background. The processes in the back office of Educateca site are also based in our work in [8, 15] where we introduce a folksonomy based approach to e-learning 2.0.

\section{References}

1. Chatti, M.A., Jarke, M., Frosch-Wilke, D.: The future of e-learning: a shift to knowledge networking and social software. International Journal of Knowledge and Learning 3(4/5), 404-420 (2007)

2. Downes, S.: e-learning 2.0. eLearn. 10 (October 2005)

3. Advanced Distributed Learning (ADL): Sharable Content Object Reference Model, SCORM 2004, 3rd edn. (2004), http: / / www . adlnet. org

4. Rogers, C.P., Liddle, S.W., Chan, P., Doxey, A., Isom, B.: Web 2.0 learning platform: Harnessing collective intelligence. Online Submission 8, 16-33 (2007),

http://www.eric.ed.gov/ERICWebPortal/detail ?accno=ED498811

5. Rey López, M., Díaz Redondo, R., Fernández Vilas, A., Pazos Arias, J., López Nores, M., García Duque, J., Gil Solla, A., Ramos Cabrer, M.: T-MAESTRO and its Authoring Tool: Using Adaptation to Integrate Entertainment into Personalized T-learning. Multimedia Tools and Applications 40(3), 409-451 (2008)

6. Fernández Vilas, J.A., Pazos Arias, J.J., Fernández Vilas, A.: Virtual Web Services: An Extension Architecture to Alleviate Open Problems in Web Services Technology, pp. 74 94. IGI Global (October 2008)

7. Fernández Vilas, J.A., Pazos Arias, J.J., Fernández Vilas, A.: VWS: Applying Virtualization Techniques to Web Services. International Journal of Computer Science and Network Security 6(5B), 120-128 (2006)

8. Rey López, M., Díaz Redondo, R., Fernández Vilas, A., Pazos Arias, J.J.: T-learning 2.0: a personalized hybrid approach based on ontologies and folksonomies. In: Computational Intelligence for Technology Enhanced Learning. Springer, Heidelberg (2010)

9. Curbera, F., Khalaf, R., Mukhi, N., Tai, S., Weerawarana, S.: The next step in Web services. Communications of the ACM 46 (October 2003)

10. Vossen, G., Westerkamp, P.: Why service-orientation could make e-learning standards obsolete. International Journal of Technology Enhanced Learning 1(1/2), 85-97 (2008)

11. Dong, B., Zheng, Q., Yang, J., Li, H., Qiao, M.: An E-learning Ecosystem Based on Cloud Computing Infrastructure. In: 9th IEEE International Conference on Advanced Learning Technologies, ICALT 2009, pp. 125-127 (July 2009)

12. Dong, B., Zheng, Q., Qiao, M., Shu, J., Yang, J.: BlueSky Cloud Framework: An ELearning Framework Embracing Cloud Computing. In: 1st International Conference on Cloud Computing (CloudCom 2009), Beijing, China (December 2009)

13. Sulistio, A., Reich, C., Dölitzscher, F.: Cloud Infrastructure \& Applications - CloudIA. In: Proceedings of the 1st International Conference on Cloud Computing (CloudCom 2009), Beijing, China, December 1-4 (2009)

14. Vouk, M., Averitt, S., Bugaev, M., Kurth, A., Peeler, A., Shaffer, H., Sills, E., Stein, S., Thompson, J.: Powered by VCL - Using Virtual Computing Laboratory (VCL). In: Proc. 2nd International Conference on Virtual Computing (ICVCI), pp. 1-10 (May 2008)

15. Rey López, M., Díaz Redondo, R., Fernández Vilas, A., Pazos Arias, J.: Use of Folksonomies in the Creation of Learning Experiences for Television. In: Upgrade. Monograph: Technology-Enhanced Learning, vol. IX(3), pp. 21-26 (June 2008) 